\title{
Prototipo computarizado de un mueble doméstico multifunción para su producción y estrategias de mantenimiento.
}

Computed prototype of a multifunction domestic furniture for production and maintenance strategies.

Wilson Alejandro Huilca Álvarez. ' , Vanessa Lorena Valverde González . ${ }^{2}$, Cesar Daniel Arregui Toro. ${ }^{3}$

Recibido: 05-08-2019 / Revisado: 15-09-209 /Aceptado: 28-09-2019/ Publicado: 04-10-2019

\begin{abstract}
.
DOI: https://doi.org/10.33262/cienciadigital.v3i4.955

There are specific multifunctional furniture to use small spaces, most of these multifunctional furniture are expensive, due to the design and the number of pieces used, remembering also, that its location in the market is not easy The object of the study is the design of a graphic prototype of a household furniture with 3 specific functions, sofa, bunk and trunk with emphasis on its possible production and preventive maintenance strategies. This design is aimed at improving the comfort of people, especially those who live in small homes. Its potential construction and commercialization is feasible, due to its economic assembly and manufacturing. It is important to remember that the usefulness of the article is aimed at complying with the manufacturing and safety requirements, so the parameters detailed in resolution 14297 have been taken into account, regarding the RTE INEN 104 "FURNITURE" regulation (Official Supplement Registry No 351, 2010) of the Republic of Ecuador. As a result, the prototype was obtained in a drawing project of 3 fixed and connected assemblies to change the mechanical position according to the need, with its respective production diagram and a maintenance plan of the multifunction furniture, for the connection catalogs, using in its construction materials manufacturing such as carpentry, textile and mechanical elements.
\end{abstract}

\footnotetext{
${ }^{1}$ Escuela Superior Politécnica de Chimborazo, Chimborazo, Ecuador, wilson.huilca@espoch.edu.ec

${ }^{2}$ Escuela Superior Politécnica de Chimborazo, Chimborazo, Ecuador, v_valverde@espoch.edu.ec

${ }^{3}$ Escuela Superior Politécnica de Chimborazo, Chimborazo, Ecuador, cesar.arregui@espoch.edu.ec
} 
Keywords: Domestic furniture, graphic prototype, production, maintenance

\section{Resumen.}

Existen muebles multifuncionales diseñados para aprovechar los espacios pequeños, la mayor parte de estos muebles multifuncionales son costosos, debido al diseño y al número de piezas utilizadas, recalcando también, que no es fácil su localización en el mercado

El objeto del estudio, es el diseño de un prototipo gráfico de un mueble del hogar con 3 funciones concretas, sofá, litera y baúl con énfasis en su posible producción y estrategias de mantenimiento preventivo. Dicho diseño está orientado a mejorar la comodidad de las personas, principalmente aquellas que habitan hogares pequeños. Su potencial construcción y comercialización es factible, debido a su económico armado y fabricación. Es importante recalcar que la utilidad del artículo está orientada a cumplir estándares de fabricación y seguridad, por lo cual se ha tomado en cuenta los parámetros detallados en la resolución 14297, respecto al reglamento RTE INEN 104 "MUEBLES" (Registro Oficial Suplemento No. 351, 2010) de la república del Ecuador.

Como resultado se obtuvo el prototipo en proyecto de dibujo de 3 ensambles fijos y conectados para cambiar de posición mecánica de acuerdo a la necesidad, con su respectivo diagrama de producción y un plan de mantenimiento del mueble multifunción, para los catálogos de comercialización, utilizando en su fabricación materiales de construcción como: elementos de carpintería, textiles y mecánicos

Palabras claves: Mueble doméstico, prototipo gráfico, producción, mantenimiento

\section{Introducción.}

El presente trabajo, tiene por objetivo mostrar gráficamente mediante un software computarizado de diseño mecánico llamado Solidworks, la forma de armado y ensamble de un mueble doméstico multifunción, con la finalidad de producirlo en serie, denotando también, las estrategias de mantenimiento del mismo con la finalidad de comercialización.

Los Sistemas de Gestión de la Producción integran las diferentes funciones de Planificación y Control de la Producción; a partir de la utilización de técnicas, diagramas, gráficos y software, que facilitan los cálculos y decisiones en torno a la selección de las mejores variantes de producción. (Tacuri 2010)

El programa de diseño en 3D SolidWorks es una ayuda y un avance tecnológico para la ingeniería donde se puede lograr diseñar productos con mayor rapidez y con medidas a 
escalas reales estas ya sean ampliadas o reducidas. Este programa de diseño en la medida que lo utilice en una empresa para mejorar sus procesos lo podrá realizar en menor tiempo y con un costo menor. (Ccapa 2019)

Respecto al mantenimiento de las partes del mueble doméstico multifunción se mostrará la secuencia gráfica de los trabajos necesarios de acuerdo con un método de mantenimiento preventivo, con el fin de garantizar el buen estado de las partes móviles y fijas del mueble doméstico multifunción, al momento que sea comercializado, para que pueda constar en los catálogos de comercialización.

En la actualidad, es indispensable generar estrategias que permitan abaratar costos cotidianos, debido altos importes de vida. En las grandes ciudades tal parece que los espacios domiciliarios cada vez son más pequeños, debido los proyectos de las firmas inmobiliarias que se adaptan a la densidad poblacional.

El crecimiento tan acelerado de las grandes ciudades unido al encarecimiento general de la vivienda va convirtiendo los hogares en sitios más y más pequeños, factores con este, evidencian un gran problema para el futuro en el mundo entero, y nuestras ciudades, en donde la falta de espacios para construir ya es evidente, teniendo en cuenta un futuro de construcción y diseño en espacios mínimos. (Homy Decoración 2017).

La industria de carpintería y del mobiliario de madera es una de las actividades más antiguas de la humanidad, y ha ido evolucionando a lo largo del tiempo a medida que se desarrollaba la actividad industrial, permitiendo, los avances tecnológicos, adaptarse a las materias primas disponibles y a las necesidades del mercado. (Bermudez 2005).

En los países en vías de desarrollo industrial producen aproximadamente el $21 \%$ del valor total de los muebles a nivel mundial, aunque su participación viene incrementándose en los últimos años por importantes inversiones destinadas a elevar la capacidad productiva dirigida principalmente a la exportación, destacando China, México y Polonia. (Apartmueble 2018).

La diversidad de la demanda y los costos inmobiliarios, han generado varias tendencias respecto a la elección de viviendas, un aspecto importante son los nuevos costos de vida en grandes urbes. Una nueva generación de apartamentos y casas pequeñas evolucionan como los productos de mayor valor añadido de las economías desarrolladas: aumenta el valor y el servicio de los espacios en los lugares más vibrantes, pero en ocasiones se reduce tanto su tamaño como la acumulación de bienes físicos en su interior. (Boullosa, 2013)

El crecimiento de la industria manufacturera de muebles del hogar, ha promovido la creación de productos propios e innovadores, en las cuales se ha fusionado materiales como madera y aluminio destacando la creación de toda una variedad de diseños nuevos los cuales han llamado la atención de los posibles compradores posicionado una buena imagen en la mente del consumidor. (Urgiles, 2016) 
Tomando en cuenta estos antecedentes, ha sido tendencia en las últimas décadas, la fabricación y comercialización de los muebles multifunción en los hogares de todas partes del mundo. Tomando como referencia los muebles tradicionales se han modificado las partes y se han articulado las funciones como por ejemplo los sofás-camas, que son un tipo de mueble que, al ser desplegados, forman una cama, empleados con la finalidad de economizar el espacio disponible. (Fresnel 2000)

La importancia de los modelos de muebles ergonómicos y confortables se ha convertido en tendencia. El diseño contemporáneo, expresa que los muebles son diseñados apropiadamente para cada espacio y los colores son elegidos en función al estilo que se desea plasmar formando a la vez una serie de elementos introducidos en el diseño. (Levante 2017).

Ya no solo es común adaptar el mobiliario del hogar al diseño arquitectónico de las viviendas, actualmente es posible adaptar muebles funcionales que sean capaces de brindar comodidad y funcionalidad a los habitantes de los hogares por ejemplo ya se han implementado por mucho tiempo los sofás camas, productos que han servido como sofá en su posición normal y tener la función de cama y de sofá reclinable. (Martínez 2017)

La historia del mueble multifunción no es reciente, pero en los últimos tiempos ha despegado su popularidad. Por ejemplo, el sofá cama es ya todo un clásico en muchos hogares. Se caracterizan por su doble función: sofá durante el día y cama para la noche. (Mejorado 2018)

El sofá cama es un mueble versátil que combina su función de asiento durante el día con el de cama durante la noche. Se usan para aprovechar el espacio disponible en habitaciones pequeñas o como mueble auxiliar para acoger invitados en momentos puntuales. (Salamea 2011)

Uno de los objetivos principales de los muebles multiuso, es congregar varias tareas en un solo objeto y satisfacer las necesidades eventuales del espacio a través de mecanismos de acción que permitan redistribuir y liberar el espacio cuando un objeto no esté en uso, pudiéndose realizar varias actividades en una misma área (Lope 2005).

Muebles multi - uso como los modelos multifuncionales Herb, han sido diseñados para brindar mucha comodidad y no solo para trabajar, este sofá es ideal para viviendas con pocos metros cuadrados, ya que tiene la ventaja de llevar incorporados algunos de los elementos con los que solemos rodear el sofá, como mesas auxiliares. (Pendones, 2018)

Los sofás cama pueden encontrarse no solo en muchos estilos sino también en varias medidas. Los más típicos son las camas para dos personas (sofás de dos/tres plazas) si bien también existen camas individuales (normalmente en sofás de una plaza). (Del Pilar, Meneses, Castro 2017).

La forma más eficiente y segura de fabricar un mueble multifunción es mediante estructuras metálicas. Una estructura metálica, es el ensamble de elementos que mantienen una forma y 
unidad. Sus objetivos son: resistir cargas resultantes de su uso y de su peso propio y darle forma a un cuerpo, obra civil o máquina. (Merino 2008).

Existen estructuras móviles, independientemente de su construcción, pueden desplazarse sin que se tenga que afectar su estructura propia. Para poder desplazar dicha estructura se emplean articulaciones. (Ofisillas 2017)

La optimización de espacios permite ahorrar recursos sin tener que renunciar al diseño y elegancia cuando se utilizan muebles multifuncionales. Por otro lado, el fácil almacenamiento del mobiliario permite combinar eficazmente los espacios y crear ambientes personalizados. (Miele, 2015).

La durabilidad del mobiliario del hogar es considerado importante, por lo cual es importante gestionar su construcción, fusionando correctamente los materiales, como estructuras metálicas, madera e implementos textiles. Entre más elementos metálicos contenga el mueble, se mejora su estructura, son ligeros, ocupan poco espacio visual y son tremendamente funcionales. (Serrano 2010)

Respecto a materiales, la madera llamada MDF, es fácil de trabajar pues viene fabricado en láminas pues eso propende a ahorrar recursos. Durante el proceso de fabricación se pueden añadir determinados productos químicos con el fin de añadir características adicionales al mdf, como repeler el agua o evitar la aparición de hongos o moho. (Maderas Santana 2015).

La sujeción de las piezas metálicas y la madera se realiza con tornillos ya que permiten anclar un objeto a otro o un elemento a un revestimiento de manera. En uniones sencillas, normalmente en madera y materiales derivados, se introduce el tornillo directamente, y son su longitud y anchura los que determinarán la sujeción que puede ejercer. (Leroy, 2016)

\section{Metodología}

Respecto a los materiales, es sensato pensar en los componentes del ensamble para la construcción del mueble multifuncional, tomando en cuenta las 3 posiciones que generan los usos de dicho mueble.

Cuadro 1. Tabla descriptiva de materiales

\begin{tabular}{cccc}
\hline Cantidad & Materiales - Descripción & Valor Unitario & Valor total \\
\hline 5 & Bloques armado de mdf reforzado $1 * 1$ & 30 & 150 \\
10 & Metros de tela & 5 & 50 \\
10 & Tornillos & 0,02 & 0,2 \\
1 & Caja de Tachuelas & 1,50 & 1,5 \\
1 & pegamento & 2 & 2 \\
3 & Pliegos de esponja & 7 & 21 \\
1 & Escalera metálica & 7,5 & 7,5 \\
\hline
\end{tabular}


Fuente. Elaboración propia

Respecto al método de investigación se puede especificar la importancia de generar un procedimiento analítico, para establecer las relaciones entre los componentes físicos del mueble y un procedimiento comparativo, debido a la importancia de tomar en cuenta estudios, proyecto y prototipos ya establecidos y/o fabricados

Respecto al método de conformación del presente estudio, se expresan 5 procedimientos básicos:
a) Idea de fabricación
b) Bosquejo,
c) Diseño del prototipo en software. Para el presente estudio se utilizó Solidworks 2018
d) Diagrama de proceso de armado del mueble
e) Plan resumido de mantenimiento

Cabe denotar que el presente estudio no llega hasta la construcción del mueble multifunción, llegando simplemente a la generación del prototipo gráfico, diseño del proceso productivo y las estrategias de mantenimiento preventivo.

Es de vital importancia tomar en cuenta datos estratégicos adicionales como:

- Tamaño de espacios regulares en hogares estándar

- Medidas antropométricas estándar

- Indagación de softwares apropiados

- Estudio de materiales (para ser representados en los dibujos)

Respecto al plan de mantenimiento

Alcance: Este procedimiento detalla paso a paso las actividades de inspección y localización de los accesorios y componentes principales y secundarios del mueble multifunción.

Riesgos involucrados: Atrapamiento, golpes y heridas en las manos.

Medidas Preventivas: Utilizar E.P.P. (elementos de protección personal gafas, zapatos de seguridad y guantes de pupos).

Herramientas y materiales necesarios para el mantenimiento preventivo. 
Tabla 2. Herramientas y materiales de mantenimiento

\begin{tabular}{cc}
\hline Ítem & Descripción \\
\hline 1 & Caja de herramientas básicas para reparación de muebles. \\
2 & Penetrante de acción rápida, libera y lubrica piezas atascadas, desplaza la \\
humedad.
\end{tabular}

Fuente. Elaboración propia

Respecto al diseño en solidworks se utilizó las herramientas de diseño y ensamble como:

- Barra de herramientas 2D a 3D

- Barra de herramientas Alinear

- Barra de herramientas Anotaciones

- Barra de herramientas Ensamblaje

- Barra de herramientas Bloques

- Barra de herramientas Curvas

- Barra de herramientas Operaciones cierre

- Barra de herramientas Operaciones

- Barra de herramientas Capa

- Barra de herramientas Herramientas de diseño

- Barra de herramientas Formato de línea

- Barra de herramientas Macro

- Barra Herramientas de moldes

- Barra de herramientas Capturas rápidas

- Barra de herramientas Geometría de referencia

- Barra de herramientas Herramientas de renderizado

- Barra de herramientas de Scanto3D

- Barra de herramientas Estándar

- Barra de herramientas Vistas estándar

- Barra de herramientas Superficies

- Barra de herramientas Tabla

- Barra de herramientas de Toolbox

- Barra Herramientas

- Barra de herramientas Ver

- Barra de herramientas Web

- Barra de herramientas Piezas soldadas 


\section{Resultados}

Respecto al dibujo mecánico y maqueta realizada.

El dibujo simulado, considera medidas estandarizadas por su ancho y largo tomando en cuenta una cama de dos plazas y tomando en cuenta personas con una medida de $1,8 \mathrm{~m}$

El ancho de la esponja, dependerá de la fabricación, en el prototipo gráfico se estima una dimensión

Las siguientes especificaciones gráficas y explicativas, podrían ser expuestas en un manual de uso, y son el resultado del presente estudio por presentar los procedimientos del armado de transformación del mueble multifunción

\section{Diseño mueble multiuso}

El diseño prototipo ha sido desarrollado como un mueble multi-uso para cumplir 3 funciones:

1. Sofá: Cumple la función de un sofá familiar de cuatro compartimientos.

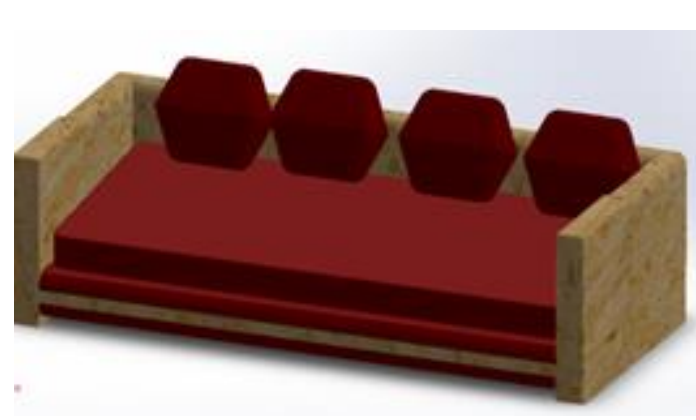

Gráfico 1. Vista frontal - función sofá Fuente. Elaboración propia

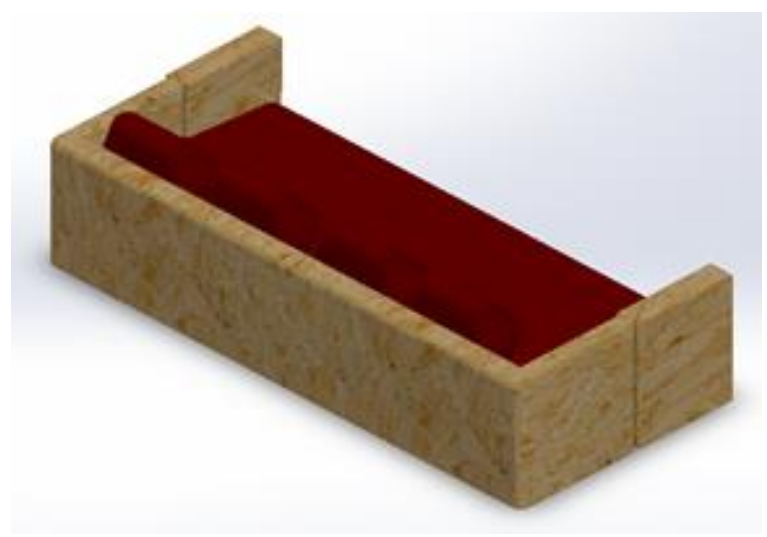

Gráfico 2. Vista lateral - función sofá

Fuente. Elaboración propia

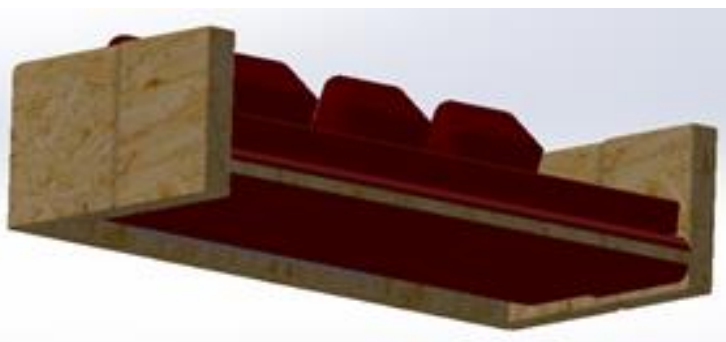

Gráfico 3. Vista posterior - función sofá Fuente. Elaboración propia
Gráfico 4. Vista inferior - función sofá Fuente. Elaboración propia 


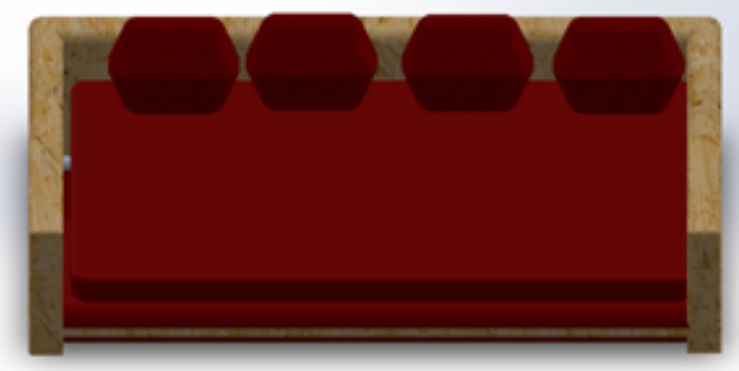

Gráfico 5. Vista superior - función sofá Fuente. Elaboración propia

2. Función: Cama litera

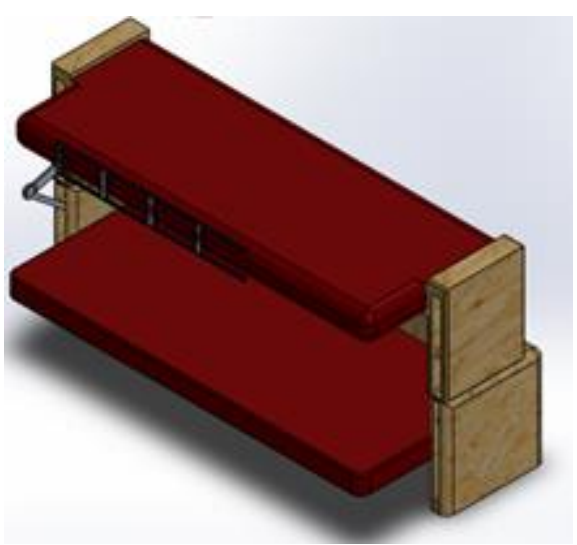

Gráfico 6. Función cama litera

Fuente. Elaboración propia

Para convertir de sofá a litera para dos personas se debe realizar los siguientes pasos:

Paso 1. Retirar las almohadillas y eleve el asiento superior en el que ese encontraba sentado.

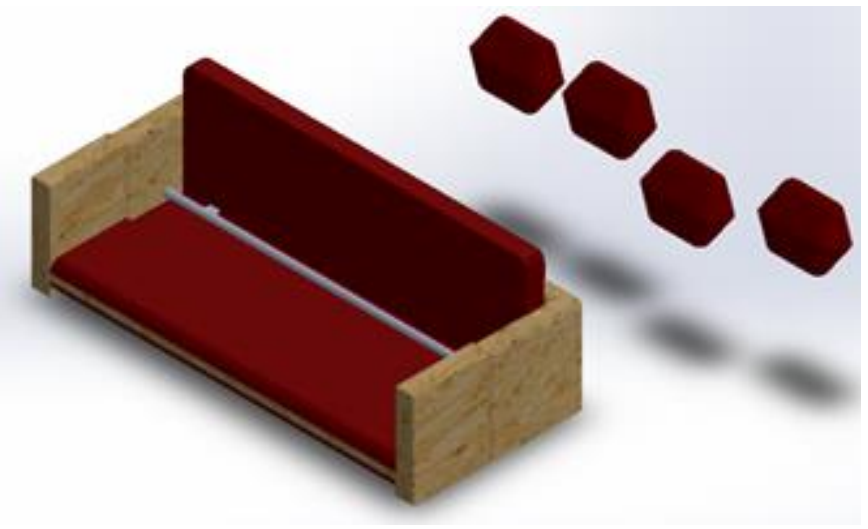

Gráfico 7. Conversión de sofá a litera. Paso 1

Fuente. Elaboración propia 
Paso 2. Sostener de un extremo del sofá, y sujetar haciendo presión hacia arriba. Las bisagras del mueble, ayudarán a que la transformación sea rápida y sencilla

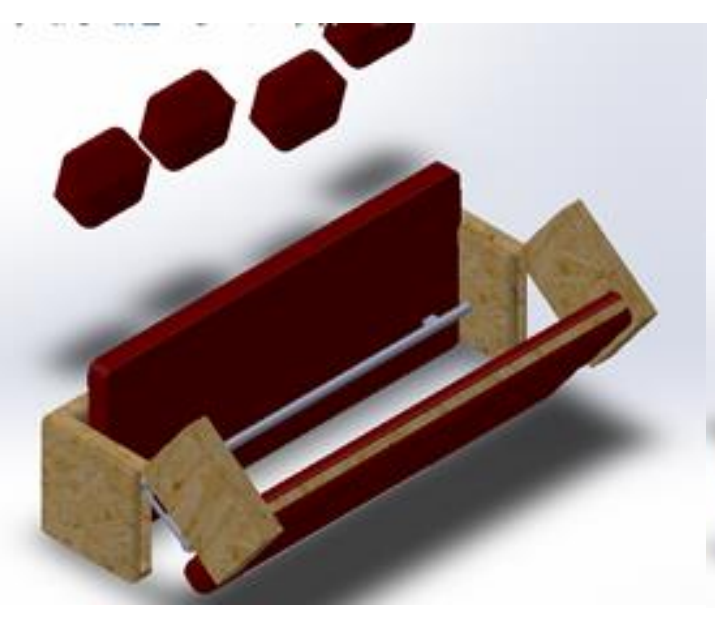

Gráfico 8. Conversión de sofá a litera.

Fuente. Elaboración propia

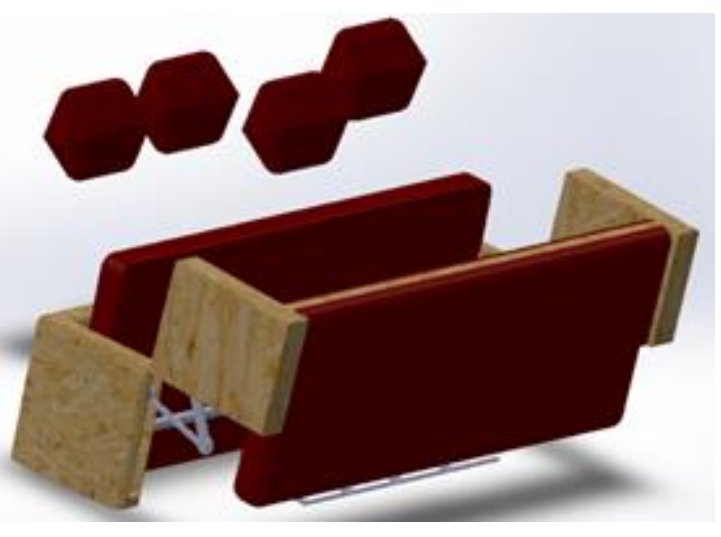

Gráfico 9. Trayectoria de elevación para el armado de la litera Fuente. Elaboración propia

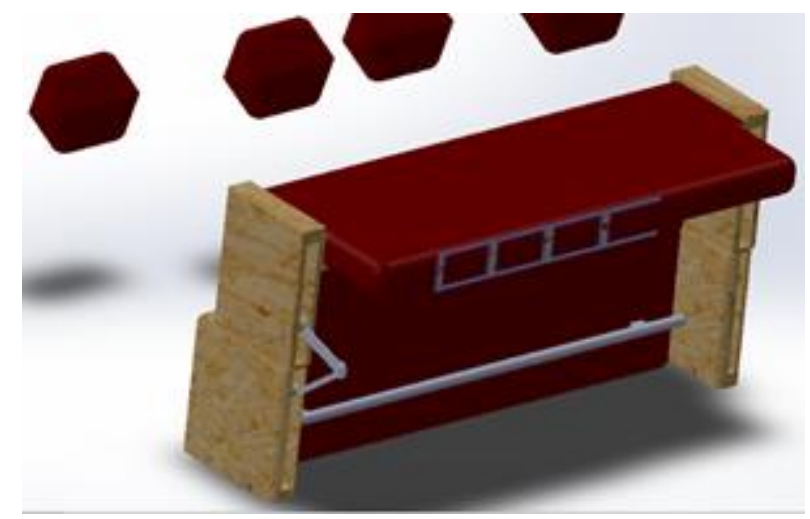

Gráfico 10. Conversión de sofá a litera. Paso 2 (trayectoria de elevación para el armado de la litera - 2)

Fuente. Elaboración propia

Paso 3. Cuando estén las dos superficies en contacto, se debe bajar la pequeña escalera que se encuentra en uno de sus laterales y además se baja nuevamente la parte del sofá que se había levantado anteriormente. 

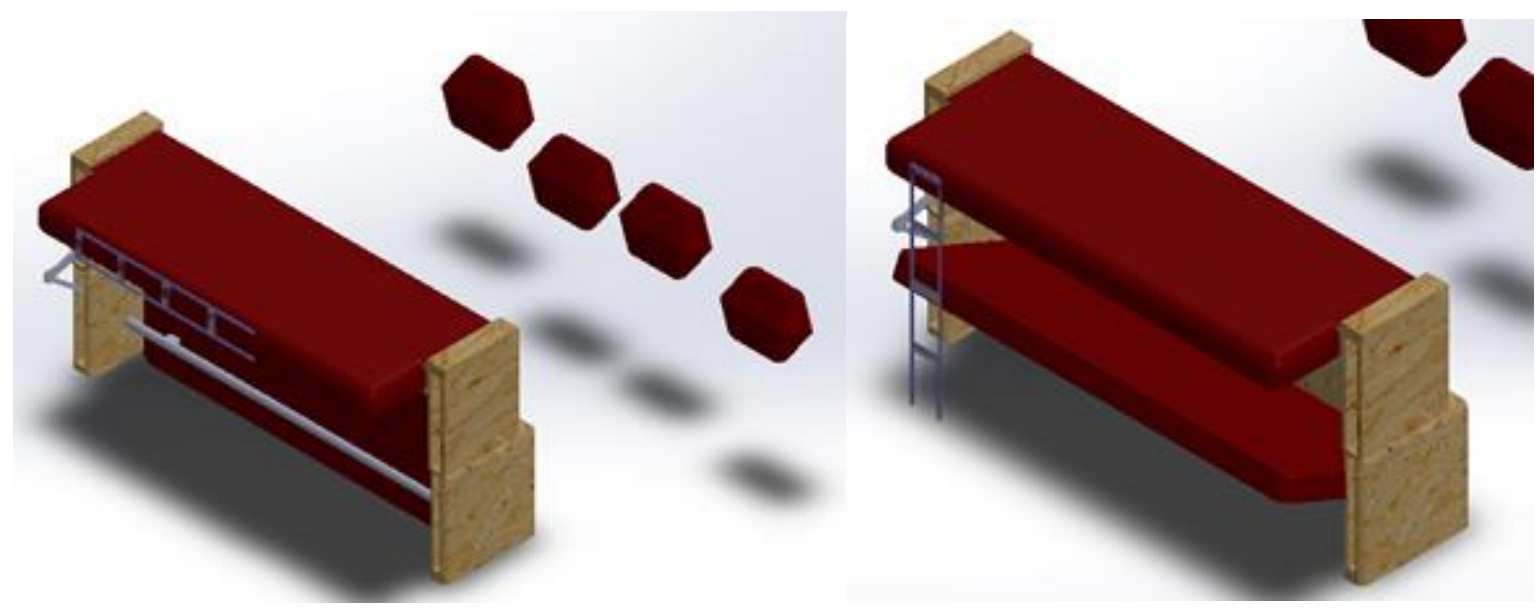

Gráfico 11. Conversión de sofá a litera. Paso 3 (Contacto de 2 superficies) Fuente. Elaboración propia
Gráfico 12. Conversión de sofá a litera. Paso 3 (Nivelación de los pisos) Fuente. Elaboración propia

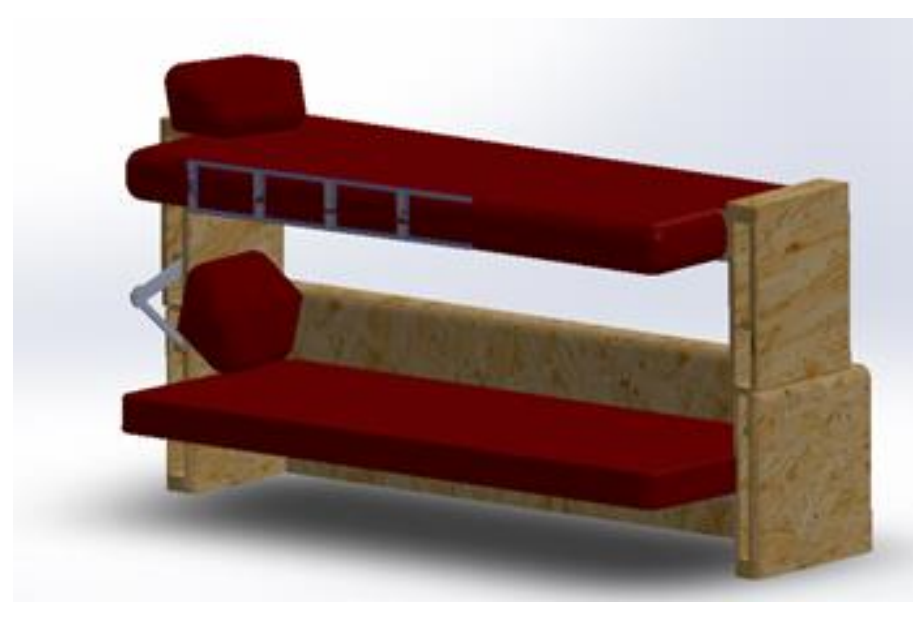

Gráfico 13. Conversión de sofá a litera.

Paso 3 (Cama litera armada con cojines, vista frontal)

Fuente. Elaboración propia

Función 3. Baúl

Paso 1. Se debe retirar las almohadillas y se debe revertir el procedimiento anterior como si se deseara transformar nuevamente a sofá. 


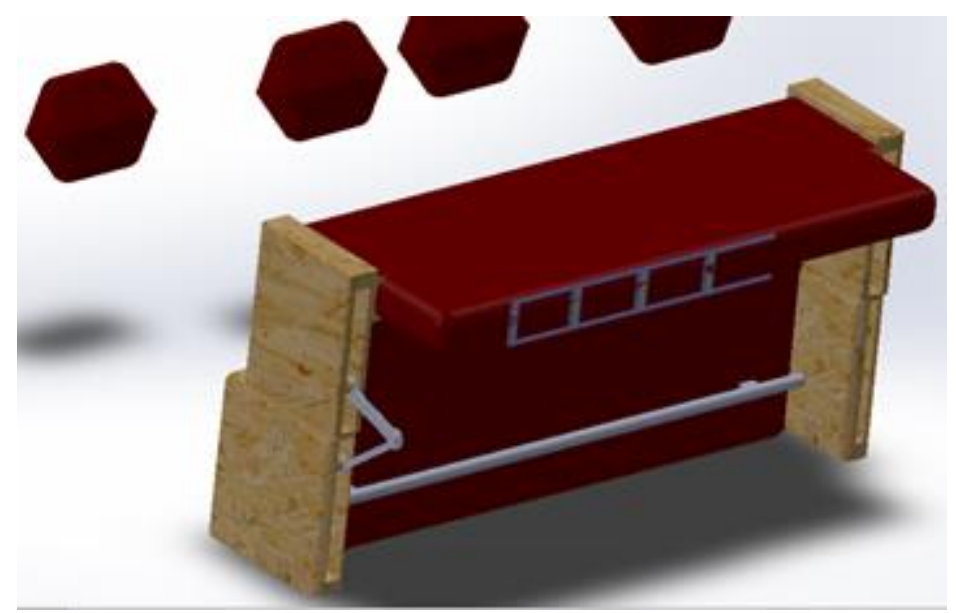

Gráfico 14. Conversión a baúl. Gráfico 5.

Conversión a baúl. (Inicio de la trayectoria de armado)

Fuente. Elaboración propia

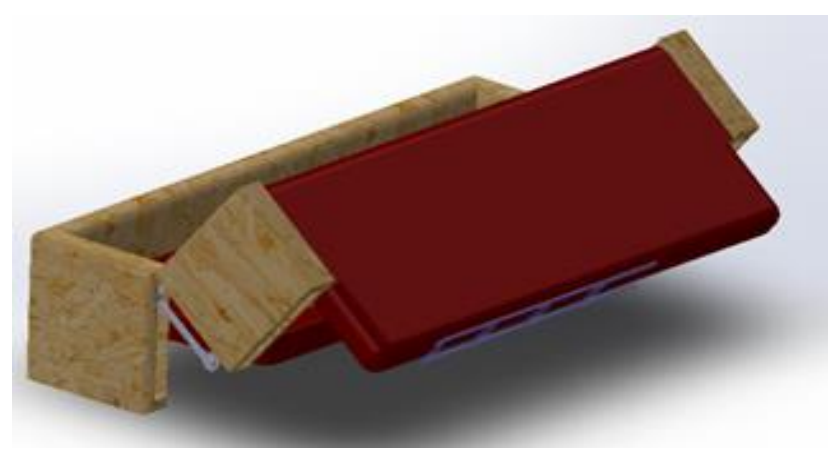

Gráfico 15. Conversión a baúl. Gráfico 5 .

Conversión a baúl. (Durante la trayectoria de armado)

Fuente. Elaboración propia

Paso 2. Descender las bases hasta que coincidan las superficies

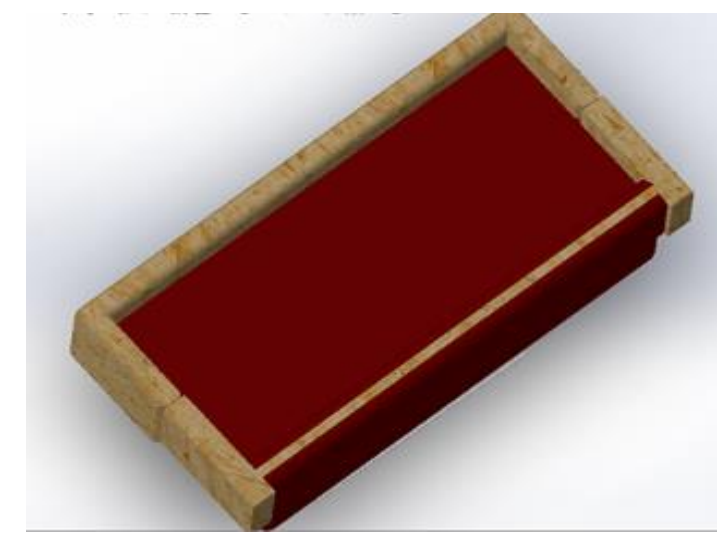

Gráfico 16. Conversión a baúl. (bases coincidentes)

Fuente. Elaboración propia 
Paso 3 Colocar una tapa en la parte superior

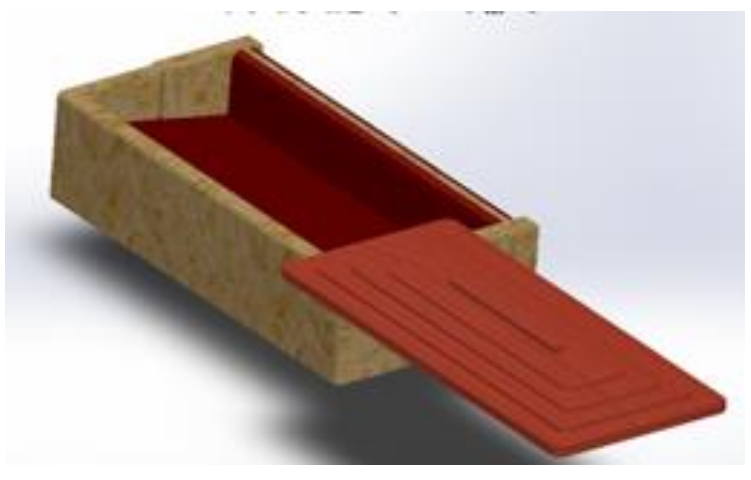

Gráfico 17. Conversión a baúl. (tapa en proyección de colocación)

Fuente. Elaboración propia

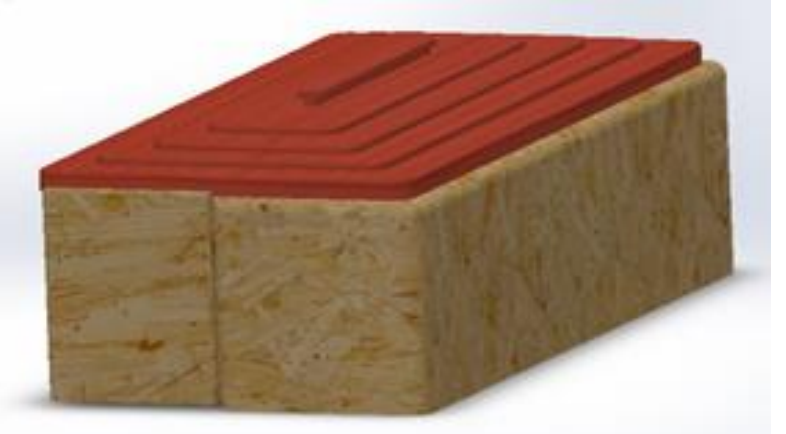

Gráfico 18. Conversión a baúl. (Baúl con tapa)

Fuente. Elaboración propia

Por ultimo este mueble se puede doblar para ocupar el menor espacio posible. Para esto elevamos la parte superior del sofá como se muestra en la siguiente figura, además giramos la parte inferior del mueble e introducimos ambas superficies entre sí.

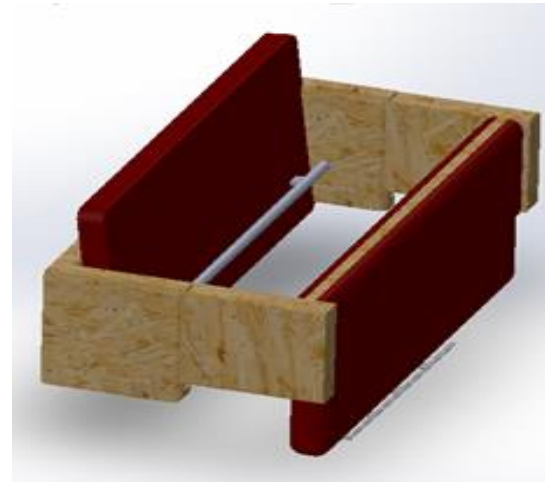

Gráfico 19. Conversión a baúl.

Fuente. Elaboración propia

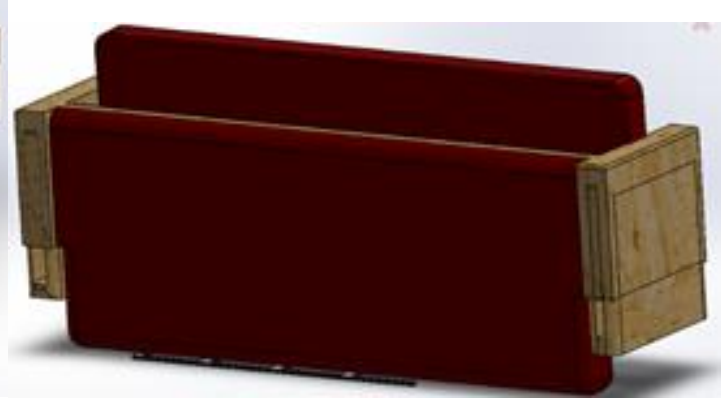

Gráfico 20. Conversión a baúl. (Mueble plegado) a baúl. (Mueble en proyección de repliegue)

Fuente. Elaboración propia 
Diagrama de proceso de producción

Se puede establecer un proceso de producción estándar para la fabricación en serie del mueble doméstico multifunción, mismo que se establece para los 3 tipos de funcionalidad del mueble.

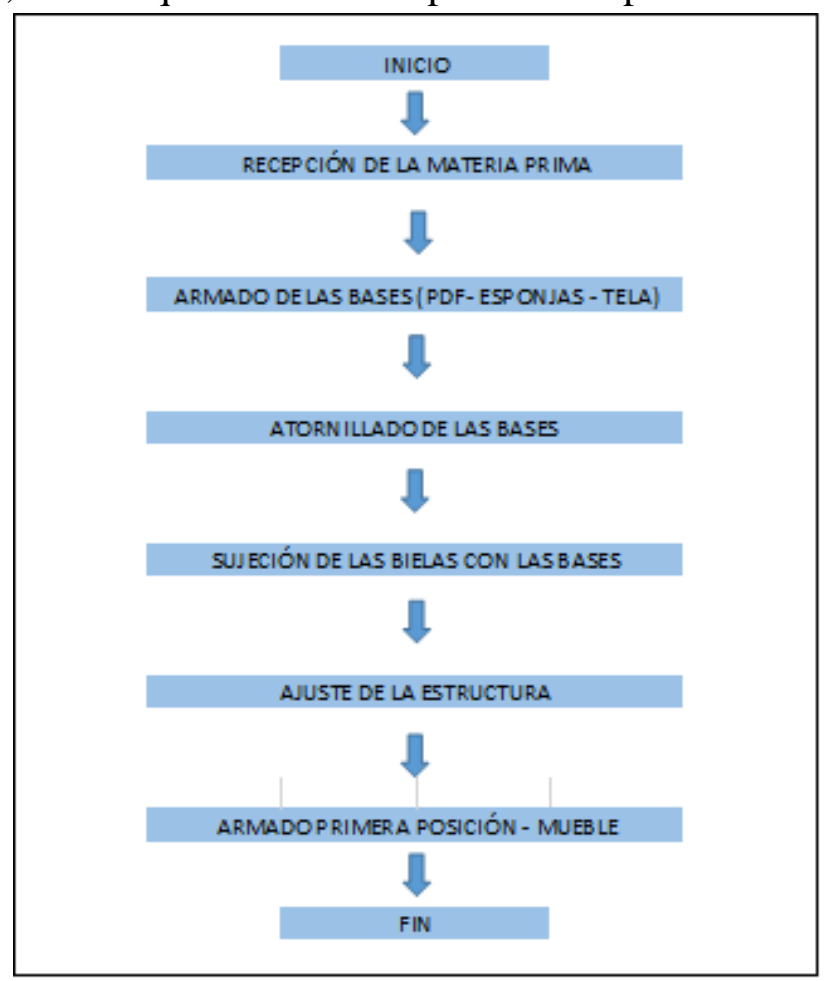

Gráfico 18. Conversión a baúl. (Baúl con tapa)

Fuente. Elaboración propia

Resultados del Plan de Mantenimiento

\section{Análisis de modos y efectos de fallos de las partes críticas del mueble multifunción}

Tabla 3. Análisis de modos y efectos de fallos

\begin{tabular}{|c|c|c|c|c|c|c|c|}
\hline $\begin{array}{l}\text { Partes del } \\
\text { mueble }\end{array}$ & Función & $\begin{array}{l}\text { Tipo de } \\
\text { función }\end{array}$ & $\begin{array}{l}\text { Falla } \\
\text { Funcional }\end{array}$ & Modo de Falla & $\begin{array}{l}\text { Efecto } \\
\text { Falla }\end{array}$ & de & Consecuencia \\
\hline $\begin{array}{l}\text { Cuerpo } \\
\text { estructural } \\
\text { del mueble }\end{array}$ & $\begin{array}{l}\text { Dar estabilidad } \\
\text { al mueble } \\
\text { multifuncional }\end{array}$ & Primaria & $\begin{array}{l}\text { No proporcionar } \\
\text { la estabilidad } \\
\text { necesaria al } \\
\text { mueble } \\
\text { multifuncional }\end{array}$ & $\begin{array}{lr}\text { Pernos } & \text { de } \\
\text { anclaje } & \text { sin } \\
\text { torque } & \\
\text { adecuado } & \end{array}$ & $\begin{array}{l}\text { Ruidos } \\
\text { holguras } \\
\text { excesivas }\end{array}$ & $\mathrm{y}$ & $\begin{array}{l}\text { Pérdida de } \\
\text { funcionalidad }\end{array}$ \\
\hline $\begin{array}{l}\text { Escalera } \\
\text { del mueble }\end{array}$ & $\begin{array}{l}\text { Conectar los } \\
\text { distintos pisos } \\
\text { del mueble } \\
\text { multifuncional }\end{array}$ & Primaria & $\begin{array}{l}\text { Incapaz de } \\
\text { soportar peso }\end{array}$ & $\begin{array}{l}\text { Conexiones } \\
\text { sueltas entre la } \\
\text { escalera y el } \\
\text { bloque armado }\end{array}$ & $\begin{array}{l}\text { Sobresfuerzo } \\
\text { por elevac } \\
\text { presión }\end{array}$ & os & $\begin{array}{l}\text { Pérdida de } \\
\text { funcionalidad }\end{array}$ \\
\hline $\begin{array}{l}\text { Bielas de } \\
\text { movilidad } \\
\text { del mueble }\end{array}$ & $\begin{array}{l}\text { Transformar el } \\
\text { movimiento } \\
\text { desde las } \\
\text { fuentes que lo } \\
\text { generan }\end{array}$ & Primaria & $\begin{array}{lr}\text { Incapaz } & \text { de } \\
\text { transformar } & \text { el } \\
\text { movimiento } & \end{array}$ & $\begin{array}{l}\text { Atascamiento } \\
\text { del mecanismo }\end{array}$ & $\begin{array}{l}\text { Sobresfuerzo } \\
\text { desgaste } \\
\text { elementos }\end{array}$ & & $\begin{array}{l}\text { Pérdida de } \\
\text { funcionalidad }\end{array}$ \\
\hline
\end{tabular}

Fuente. Elaboración propia 
Programa de mantenimiento del mueble doméstico multifunción

Tabla 4. Actividades de mantenimiento preventivo

\begin{tabular}{|c|c|c|c|c|c|}
\hline Ítem & Conjunto & $\begin{array}{c}\text { Tarea } \\
\mathbf{N}^{\circ}\end{array}$ & $\begin{array}{c}\text { Reporte } \\
\text { Fotográfico }\end{array}$ & Tareas de mantenimiento & $\begin{array}{c}\text { Tiempo } \\
\text { estimado } \\
\text { (horas) }\end{array}$ \\
\hline & & 1 & & \begin{tabular}{|c|} 
Verificación estado y/o \\
reemplazo de pernos y tuercas
\end{tabular} & 0.5 \\
\hline 1 & Estructura & 2 & & \begin{tabular}{|c|} 
Verificación, del torque \\
adecuado de las tuercas de la \\
estructura
\end{tabular} & 0.25 \\
\hline 2 & Escalera metálica & 1 & & $\begin{array}{l}\text { Revisión de estado de la } \\
\text { escalera metálica }\end{array}$ & 0.25 \\
\hline \multirow[b]{3}{*}{3} & \multirow[b]{3}{*}{$\begin{array}{l}\text { Bielas de } \\
\text { movilidad }\end{array}$} & \multirow[b]{3}{*}{3} & & $\begin{array}{l}\text { Verificación de estado de } \\
\text { palanca y acoplamientos }\end{array}$ & 0.25 \\
\hline & & & & $\begin{array}{c}\text { Revisión de desgaste de la } \\
\text { rosca }\end{array}$ & 0.25 \\
\hline & & & & $\begin{array}{c}\text { Verter fluido de limpieza y } \\
\text { lubricación de partes móviles }\end{array}$ & 0.50 \\
\hline 4 & $\begin{array}{l}\text { Bloque armado } \\
\text { de MDF } \\
\text { reforzado } 1 * 1\end{array}$ & 4 & & $\begin{array}{c}\text { Revisión de estado del bloque } \\
\text { reforzad, examine las } \\
\text { superficies de unión }\end{array}$ & 0.25 \\
\hline
\end{tabular}

Fuente. Elaboración propia

\section{Discusión de resultados}

Como se puede apreciar en cada transformación de función, los procesos de cambio son prácticos y no ameritan mayor aplicación de fuerza.

Respecto la modalidad de sofá, es fácil apreciar que la percepción de la comodidad en el proyecto de dibujo, es apreciable, la altura de los cojines y el piso es modificable en la fabricación de acuerdo al lugar en donde se comercialice.

Cuando se realiza la conversión de sofá a litera, se observa que la maniobrabilidad de los brazos en de vital importancia, ya que de ellos depende la precisión de la posición. El ajuste de las bisagras 
y los tornillos, es mediante fijación manual, pudiendo ser cambiables, pero sin dejar que se descuadren los pisos por movimientos fuertes

Las literas no tienen mucha diferencia de altura, una respecto a la otra, debido a que, si se coloca brazos demasiado grandes, se complicaría la transformación a las demás funciones. La escalera plegable por obvias razones es móvil, de hecho, está diseñada para desensamblarse

La utilidad de la función Baúl, es menos relevante, pero de uso efectivo, la tapa incorporada puede fabricarse de madera o mdf. Como se observa en los gráficos cuando los pisos se pliegan, da como resultado en una de las caras un contorno hueco útil para guardar objetos

Si un mueble multifuncional no es fácil de mover o replegar no cumple con la principal de las características, que es la simplicidad de uso. Por ello se observa que el mueble multifuncional es fácil de guardar y replegar para su movilización

Respecto al plan de mantenimiento de este mueble multifunción se analizó los modos de fallo y los efectos del fallo más comunes al cual se asignó una tarea de mantenimiento con una frecuencia de inspecciones trimestrales y semestrales, el enfoque del plan es crear estrategias de mantenimiento que mitiguen los modos de fallo e incrementen la vida útil de los componentes del ensamble del mueble multifunción.

\section{Conclusiones.}

- Es importante recalcar que los muebles multifuncionales, pueden carecer de ciertos detalles como elegancia o diseños rimbombantes, pero son muy prácticos al momento de economizar espacio

- Los diseños gráficos del presente estudio, son prototipos propuestos, la modificación de ciertas partes al momento de construir pueden ocurrir debido a la resistencia de algún material o alguna proporción adicional.

- El ensamble de las diferentes partes del mueble, son diseñadas para su producción y posible armado en serie en una planta de producción.

- Se puede concluir que para establecer mecanismos de industrialización antes de su construcción, se debería estandarizar procesos de calidad al momento del ensamble

- Debido a que las piezas son independientes, se puede generar actividades de proceso para su ensamble, incluso cada pieza independiente puede ser elaborada en una zona específica de fabricación

- El mantenimiento programado que se realiza es importante a la hora de realizar un acuerdo comercial para los potenciales compradores, recalcando que el plan de mantenimiento es de carácter preventivo

- Para ensamblar el producto, no se especifica en que función se debería iniciar, ya que cada pieza es flexible transformarse en moverse de acuerdo a la función deseada 
- Cabe recalcar que el presente estudio es la primera fase del prototipo, ya que en una segunda fase se fabricaría el prototipo a escala para determinar errores en el armado y ensamble de cada pieza.

\section{Referencias bibliográficas.}

Apartmueble. (26 de Enero de 2018). Sofás cama otra forma de descanso. Tarragona España. Apartmueble. Obtenido de https://apartmueble.com/es/blog/-b116.html

Bermudez, A. J. (2005). Análisis comparativo de preferencias y consumo del cliente entre muebles importados y muebles nacionales, Caso artesano del mueble de la parroquia Atahualpa provincia de Santa Elena. Santa Elena Ecuador. Obtenido de: http://repositorio.ug.edu.ec/bitstream/redug/1899/1/Garnica\%20Jarr\%C3\%ADn\%20Liset te.pdf

Boullosa N. (2013). Muebles transformables que combinan usos en espacios mínimos. Barcelona España. Fair Companies. Obtenido del sitio web: https://faircompanies.com/articles/muebles-transformables-que-combinan-usos-enespacios-minimos/

Ccapa, O. (2019). Diseño de una herramienta mecánica para reducir el tiempo de cambio de neumático averiado en camiones volquete en proyectos de movimiento de tierra. Recuperado de: http://repositorio.utp.edu.pe/bitstream/UTP/2056/1/Omar\%20Ccapa_Tesis_Titulo\%20Pr ofesional_2019.pdf

Del Pilar N, Meneses N, Castro P. (2017). Estudio de prefactibilidad para la creación de una empresa de muebles multifuncionales. Corporación Universitaria Mundo de Dios. Bogotá Colombia. Recuperado de https://repository.uniminuto.edu/handle/10656/6597?show=full

Fresnel, A. (2000). Optimización de las condiciones de corte en el maquinado de madera. Valdivia Chile. Estimación del desgaste del elemento de corte en fresado. Obtenido de sitio web: http://mingaonline.uach.cl/pdf/bosque/v21n2/art10.pdf

Homy Decoración.. (4 de octubre de 2017). Muebles multifuncionales. Vizcaya España .Obtenido de https://www.homy.es/blog/muebles-multifuncionales

Leroy. (2016). Para qué sirve un tornillo. Madrid España. Leroy Merlín. Obtenido del sitio web: http://www.leroymerlin.es/ideas-y-consejos/comoHacerlo/elegir-el-tornilloadecuado.html 
Levante. (2017). Gana metros en casa con trucos que evitan las obras. Valencia España. El Mercantil Valenciano. Obtenido del sitio web https://www.levante-emv.com/vida-yestilo/decoracion/2017/02/02/gana-metros-casa-trucos-evitan/1523773.html

Lopez, W (2005). Scribd. PLan Estratégico Tablitas. Obtenido de https://es.scribd.com/document/107137093/Plan-Estrategico-Tablitas

Maderas Santana (2015). Características de los tableros o madera. Tenerife Srgentina. Obtenido del sitio web : https://www.maderassantana.com/caracteristicas-tableros-madera-mdf/

Martínez Ros, P. (2017). Diseño de un sofá multifuncional. Repositorio Universidad Jaume. Castellón de la Plana. Recuperado de: http://hdl.handle.net/10234/173694

Mejorado G. (2018). Muebles básicos para toda la casa. Santiago de Chile. About Español

Obtenido del sitio web: https://www.aboutespanol.com/18-muebles-basicos-para-toda-la-casa2441527

Merino, J. (2008). La comodidad de un hogar domótico. Barcelona España. 20 Minutos. Obtenido del sitio web: https://www.20minutos.es/noticia/355427/0/comodidad/hogar/domotico/

Miele. (2015). Ahorra espacio en tu vivienda. Consejos prácticosObtenido del sitio web: http://www2.miele.es/mieleparati/ver_consejo.asp?id=24

Ofisillas. (2017). Los usos y ventajas de una mesa auxiliar plegable. Vizcaya España. Obtenido del sitio web: https://www.ofisillas.es/blog/ventajas-mesa-auxiliar-plegable

Pendones, C. (2018). Sofá multifuncional con zonas de trabajo. Madrid España. Decora Trix. Obtenido del sitio web: https://decoratrix.com/herb-un-sofa-multifuncional

SALAMEA, F. T. (2011). Diseño de Mobiliario Multifuncional. Universidad de Cuenca Facultad de Artes. Cuenca Ecuador.. Obtenido de http://dspace.ucuenca.edu.ec/bitstream/123456789/392/1/tesis..pdf

Serrano, A. (2010). Estructuras Metálicas. Blog Homy. Obtenido del sitio web: https://www.sodimac.cl/static/Homy/html/blog-Homy/tendencias/las-estructurasmetalicas-la-llevan.html

Tacuri, C. (2010). Propuesta para implementar un modelo de planeación y control de la producción en la empresa de muebles El Carrusel Cía. Ltda (Bachelor's thesis).

Urgiles, R. (2016). Creación y gestión de marca para la comercialización de muebles multiuso en la ciudad de Machala. Obtenido del sitio web: http://www.bibliotecasdelecuador.com/Record/oai:oai:repositorio.utmachala.edu.ec:4800 0-9587/Description 


\section{PARA CITAR EL ARTÍCULO INDEXADO.}

Huilca Álvarez, W. A., Valverde González, V. L., \& Arregui Toro, C. D. (2019). Prototipo computarizado de un mueble doméstico multifunción para su producción y estrategias de mantenimiento. Ciencia Digital, 3(4), 189-209. https://doi.org/10.33262/cienciadigital.v3i4.955

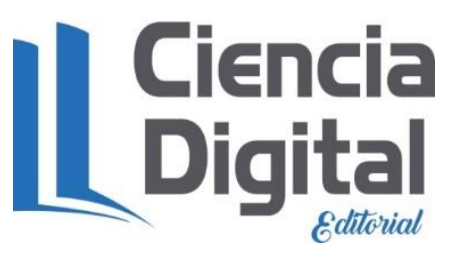

El artículo que se publica es de exclusiva responsabilidad de los autores y no necesariamente reflejan el pensamiento de la Revista Ciencia Digital.

El artículo queda en propiedad de la revista y, por tanto, su publicación parcial y/o total en otro medio tiene que ser autorizado por el director de la Revista Ciencia Digital.
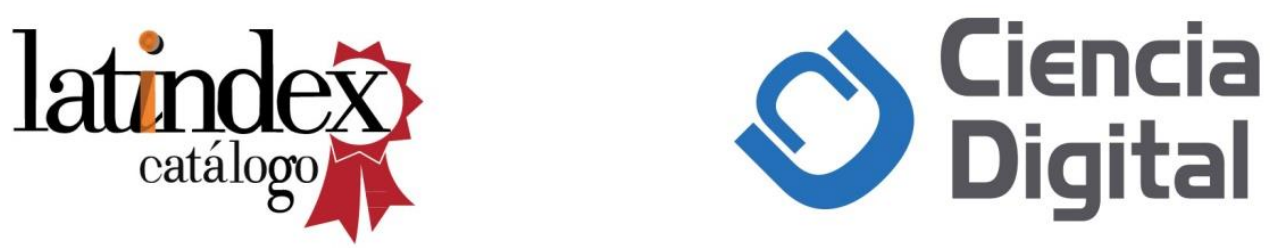